\title{
A terapia do riso em ação no Oeste do Paraná, um relato de experiência do projeto
}

\section{Alegromicicina}

Laughter therapy in action in western Paraná, an experience report from the Allegromycin project Risoterapia en acción en el oeste de Paraná, un relato de experiencia del proyecto Allegromycin

Bruna Fernandes

ORCID: https://orcid.org/0000-0002-2975-4004 Universidade Federal do Paraná, Brasil E-mail: bruufernaandes96@gmail.com

Bruno Henrique Pazza Pereira ORCID: https://orcid.org/0000-0001-9666-3228 Universidade Federal do Paraná, Brasil E-mail: brunohenriquepp@hotmail.com

Carla Roberta Mariano da Silva ORCID: https://orcid.org/0000-0001-8803-9193 Universidade Federal do Paraná, Brasil E-mail: croberta9@gmail.com

Anna Luiza Maffessoni

ORCID: https://orcid.org/0000-0002-5826-1778 Universidade Federal do Paraná, Brasil E-mail: anna.maffessoni@gmail.com

Larissa Panatta

ORCID: https://orcid.org/0000-0001-9746-1337 Universidade Federal do Paraná, Brasil E-mail: larissapanatta95@gmail.com

Bruna Orso

ORCID: https://orcid.org/0000-0003-0903-4007 Universidade Federal do Paraná, Brasil

E-mail: Brunaorso18@gmail.com

Anna Bárbara Leal Marcolin

ORCID: https://orcid.org/0000-0002-5408-2246 Universidade Federal do Paraná, Brasil

E-mail: annablm2016@gmail.com

Wemerson Geraldo de Queiroz Filho

ORCID: https://orcid.org/0000-0003-1371-7839 Universidade Federal do Paraná, Brasil E-mail: wemersonfilho84@gmail.com

Bruna Spironello Anschau

ORCID: https://orcid.org/0000-0001-6652-6062 Universidade Federal do Paraná, Brasil E-mail: brunaanschau@ufpr.br

Alessandra da Costa

ORCID: https://orcid.org/0000-0003-2840-7489 Universidade Federal do Paraná, Brasil

E-mail: alessandradacosta97@gmail.com Leonardo de Freitas Cardoso

ORCID: https://orcid.org/0000-0003-1416-3049 Universidade Federal do Paraná, Brasil

E-mail: leonardo_freitascardoso@hotmail.com Eluan Joel Rodrigues da Silva

ORCID: https://orcid.org/0000-0003-3677-4369 Universidade Federal do Paraná, Brasil E-mail: eluan.jrs@gmail.com Isadora Maria Pilati Campos

ORCID: https://orcid.org/0000-0001-5264-1836 Universidade Federal do Paraná, Brasil E-mail: isapilati@hotmail.com Amanda Rodrigues de Souza

ORCID: https://orcid.org/0000-0002-6307-5830 Universidade Federal do Paraná, Brasi

E-mail: amanda.rodriguesdesouza@ufpr.br 


\author{
Thiago Rafael Mazzarollo \\ ORCID: https://orcid.org/0000-0002-8542-8553 \\ Universidade Federal do Paraná, Brasil \\ E-mail: thiago.mazzarollo@ufpr.br \\ Jessica Cristina Ruths \\ ORCID: https://orcid.org/0000-0002-7400-1191 \\ Universidade Federal do Paraná, Brasil \\ E-mail: ruthsjessica@ufpr.br \\ Ana Carla Zarpelon Schutz \\ ORCID: https://orcid.org/0000-0002-8320-3633 \\ Universidade Federal do Paraná, Brasil \\ E-mail: ana.zarpelon@ufpr.br
}

\begin{abstract}
Resumo
Objetivo: Relatar a experiência de acadêmicos do curso de medicina no projeto de extensão "Construção de um elo entre a universidade e a comunidade: implantação do grupo Alegromicina na cidade de Toledo - Paraná." Metodologia: Trata-se de um estudo descritivo exploratório de abordagem qualitativa do tipo relato de experiência sobre um projeto de extensão da Universidade Federal do Paraná no qual se realizou visitas a pacientes internados em um hospital, nas alas clínica e pediátrica e uma clínica de hemodiálise de Toledo, Paraná, entre os anos de 2017 a 2020. Os alunos trajavam-se de palhaços para a realização das visitas que preconizavam o diálogo, abordagens musicais, atividades na brinquedoteca do hospital ou mesmo nos leitos, adequando-se ao contexto e público abordado. Resultados: As ações realizadas pelo projeto atingiram em torno de 800 pessoas por ano e ao incentivar a alegria e o alívio, diminuíram o sofrimento no contexto de fragilidade emocional vivenciada pelos pacientes, acompanhantes e até profissionais de saúde. Assim, a experiência negativa relacionada à hospitalização também foi reduzida. A capacidade de perceber o paciente para além da sua enfermidade foi um dos aspectos positivos percebidos pelos discentes que puderam experienciar humanização e a integralidade do cuidado. Conclusão: O projeto Alegromicina, trouxe benefícios aos pacientes, acompanhantes, profissionais da saúde e os alunos participantes ao promover a humanização do ambiente hospitalar. A implantação de programas que utilizam o papel do palhaço e a realização de atividades lúdicas auxiliam no prognóstico das doenças e tornam a experiência da hospitalização menos traumática.

Palavras-chave: Terapia do riso; Jogos e brinquedos; Humanização da assistência; Relações médico-paciente.
\end{abstract}

\begin{abstract}
Objective: To report the experience of medical students in the extension project "Building a link between the university and the community: deployment of the Alegromicina group in the city of Toledo - Paraná." Methodology: This is a descriptive-exploratory study with a qualitative approach, an experience report about an extension project at the Federal University of Paraná in which visits were made to patients admitted to a hospital, in the clinical and pediatric wings, and a hemodialysis clinic in Toledo, Paraná, between the years 2017 to 2020. The students dressed like clowns to carry out visits that advocated dialogue, musical approaches, activities in the hospital playroom or even in the beds, adapting to the context and audience approached. Result: The actions carried out by the project reached around 800 people per year and, by encouraging joy and relief, they reduced suffering in the context of emotional fragility experienced by patients, companions and even health professionals. Thus, the negative experience related to hospitalization was also reduced. The ability to perceive the patient beyond his illness was one of the positive aspects noticed by the students who were able to experience humanization and comprehensive care. Conclusion: The Alegromicina project brought benefits to patients, companions, health professionals and students participating in the project by promoting the humanization of the hospital environment The deployment of programs that use the role of the clown and the performance of playful activities help in the prognosis of diseases and make the hospitalization experience less traumatic.
\end{abstract}

Keywords: Laughter therapy; Play and playthings; Humanization of assistance; Physician-patient relations.

\title{
Resumen
}

Objetivo: Dar a conocer la experiencia de los estudiantes de medicina en el proyecto de extensión "Construyendo un vínculo entre la universidad y la comunidad: implantación del grupo Alegromicina en la ciudad de Toledo - Paraná". Metodología: Se trata de un estudio descriptivo-exploratorio con abordaje cualitativo, como un relato de experiencia sobre un proyecto de extensión en la Universidad Federal de Paraná en el que se realizaron visitas a pacientes ingresados en un hospital, en las alas clínica y pediátrica, y un Clínica de hemodiálisis en Toledo, Paraná, entre los años 2017 a 2020. Los alumnos se vistieron de payasos para realizar visitas que propugnaban el diálogo, planteamientos musicales, actividades en la ludoteca del hospital o incluso en las camas, adaptándose al contexto y público al que se acercaba. Resultado: Las acciones llevadas a cabo por el proyecto alcanzaron alrededor de 800 personas al año y, al fomentar la alegría y el alivio, redujeron el sufrimiento en el contexto de fragilidad emocional vivida por pacientes, acompañantes e incluso profesionales de la salud. Así, también se redujo la experiencia negativa relacionada con la hospitalización. La capacidad de percibir al paciente más allá de su enfermedad fue uno de los aspectos positivos percibidos por los estudiantes que pudieron experimentar la humanización y la atención integral. 
Conclusión: El proyecto Alegromicina trajo beneficios a los pacientes, acompañantes, profesionales de la salud y estudiantes participantes del proyecto al promover la humanización del entorno hospitalario La implementación de programas que utilizan el papel del payaso y la realización de actividades lúdicas ayudan en el pronóstico de enfermedades y hacer que la experiencia de hospitalización sea menos traumática.

Palabras clave: Risaterapia; Juego e implementos de juego; Humanización de la atención; Relaciones médicopaciente.

\section{Introdução}

De Hipócrates aos dias atuais, a relação médico-paciente modificou-se constantemente, assumindo a realidade dos conhecimentos à época. No século XVIII, com o surgimento dos hospitais e a evolução do conhecimento anatômico, microbiológico e técnico da medicina permitiu a distinção entre os termos sintoma e doença (Dario, 2019). Desse modo, uma nova medicina surge, com o foco médico centrado apenas no diagnóstico e tratamento das enfermidades (Dario, 2019). O obsoleto modelo evidenciava o médico como o centro da relação interpessoal ao considerar que era o único detentor do conhecimento, assumindo a responsabilidade total da tomada de decisões. Desconsiderava também a comunicação com o paciente e a participação deste, desvalorizando-o e tornando-o dependente e passivo (Lopes \& Dias, 2019). Esse cenário foi se alterando ao longo do tempo em busca de uma visão mais humanizada do serviço de saúde e uma atenção mais voltada à integralidade do paciente. Destacou-se também a necessidade de amenizar o ambiente do cuidado, tendo a figura do palhaço representado um marco importante desse processo de humanização.

O palhaço, com registros desde 2500 a.C., acumulou diversas mudanças na característica e na atuação durante a sua história (Beraldi, 2011). Os primeiros relatos referem-se no Antigo Egito, onde ocorriam apresentações do "bobo da corte" a fim de divertir o Faraó e a sociedade em geral (Beraldi, 2011). O circo moderno apresenta Philip Astley como o seu grande "inventor" e "criador". Juntamente a saltimbancos, acrobatas e cavaleiros, nos circos de 1768, já figuravam os palhaços (Pines Jr., Silva, Lazier, Gonçalves \& Leão Jr., 2013). Uma das expansões da atividade do palhaço foi para o ambiente hospitalar, onde há uma maior utilização da improvisação, associado à música, dança, técnicas de recreação e de interpretação (Beraldi, 2011).

O objetivo do palhaço na vivência hospitalar é a desconstrução de uma visão apenas negativa do hospital e sua ressignificação como um local em que é possível existir brincadeiras, humor, riso, imaginação, arte e criatividade. O pioneirismo de um palhaço norte-americano em performar em um hospital no "Dia do Coração", abriu as portas da medicina para o reconhecimento e aceitação da terapia do riso (Crockett, 2015). Michael Christensen, cofundador da Big Apple Circus Clown Care Unit, explorou a ambientalização do palhaço em áreas médicas ao criar uma divisão especializada em performances hospitalares com palhaços treinados em habilidades de circo e improvisação, sem violar as normas de paramentação e higienes que o local exige (Crockett, 2015). O médico "Patch" Adams foi um dos entusiastas da terapia do riso e da presença e personificação do palhaço nos hospitais, após perceber quão falho é o contato, a cumplicidade e a compaixão no meio hospitalar (Adams \& Mylander, 2002; Luchesi \& Cardoso, 2012; Luiz, 2007). No Brasil, o expoente desta iniciativa foi o besteirologista Wellington Nogueira, de codinome Dr. Zinho, que possibilitou a criação do projeto "Doutores da Alegria" e assim abriu caminho para o surgimento de cada vez mais grupos de palhaços hospitalares ao redor do país (Masseti, 2005).

A implantação de programas que buscam o bem-estar, através de atividades lúdicas assegura saúde à população ao despertar emoções, vínculos interpessoais, melhorar o desempenho físico-motor, o nível linguístico e a formação moral, principalmente nas crianças (Martins et al., 2020; Rodrigues, Simões \& Prodocimo, 2019). Além disso, asseguram saúde à população por meio do relaxamento da musculatura com melhora da respiração, aumento do limiar de dor, aumento da tolerância e redução da liberação de hormônios estressores (Doğan, 2020). A risada diminui o estresse e promove a liberação do hormônio endorfina, melhorando as respostas imunológicas e amenizando os impactos da hospitalização e tratamento, pois reduz as consequências das exposições a estímulos dolorosos e desagradáveis e aumenta as expectativas positivas em relação 
ao tratamento e retorno às atividades diárias, sendo um coadjuvante do tratamento médico formal (Heidari, Borujeni, Rezaei, Abyaneh \& Heidari, 2020; Mussa \& Malerbi, 2008).

A hospitalização tende a tornar-se desagradável e despertar medo e angústias para grande parte dos indivíduos, exige mudanças nos seus hábitos de vida, bem como o distanciamento de familiares, amigos e objetos pessoais (Jannuzzi \& Cintra, 2006). Para a criança, essa experiência costuma ser ainda mais traumática, uma vez que, além da modificação da sua rotina diária, com um ambiente físico muitas vezes impróprio para a realização de brincadeiras, são submetidas a diversos procedimentos desconfortáveis (Jansen, Santos \& Favero, 2010).

Considerando-se a importância da humanização e integralidade do atendimento, o presente relato de experiência teve por objetivo descrever as ações do Projeto de extensão acadêmica denominado Alegromicina e complementar a literatura atual sobre a temática da terapia do riso em ambientes hospitalares, além de inspirar novos projetos pelo país.

\section{Metodologia}

Trata-se um estudo descritivo exploratório (Nunes, Nascimento \& Luz, 2016) de abordagem qualitativa (Pereira, Shitsuka, Parreira \& Shitsuka, 2018), do tipo relato de experiência sobre o projeto de extensão "Construção de um elo entre a universidade e a comunidade: implantação do grupo Alegromicina na cidade de Toledo - PR", realizado por acadêmicos da Universidade Federal do Paraná, Campus Toledo. O público alvo do projeto foram os pacientes internados em um hospital de médio porte, onde ocorreu a fase inicial das atividades em 2017. O campo de ação foi estendido em abril de 2019 para englobar o setor de diálise de outra instituição, também em Toledo.

O nome do projeto trata-se de uma união da palavra "alegria" com o sufixo "micina", utilizado em diversos antibióticos, a exemplo de neomicina e gentamicina, o que sugere a alegria como uma forma de medicação. Este projeto é o primeiro da regional de saúde a contar com um grupo de palhaços propriamente dito, até então havia apenas iniciativas de escoteiros e outros projetos, com objetivos diferentes, dos cursos de pedagogia e psicologia de outras universidades de Toledo, que inclusive se mantiveram paralelamente, possibilitando a troca de experiências entre os grupos.

As ações iniciaram em novembro de 2017 e tiveram duração até agosto de 2020. Em março de 2018 foi realizado o I Curso de Teatro em Stanislaviski, para capacitação dos extensionistas. O projeto contou com 17 participantes que atuaram divididos em três equipes de cinco a seis extensionistas para a realização das atividades presenciais. Em todo período de execução do projeto, cinco alunos entre os voluntários receberam o recurso de bolsa de projeto de extensão pela UFPR, de acordo com classificação avaliativa determinada pelos coordenadores. Nesse período, foram realizadas visitas quinzenais de forma rotativa a pacientes que se encontravam internados nas alas clínicas e pediátricas do hospital e àqueles submetidos à diálise na clínica participante do projeto buscando, assim, abranger um público amplo.

Durante a execução do projeto, objetivou-se fornecer condições que permitissem o aprendizado e o exercício da humanização na relação entre estudantes e pacientes; promover e ampliar as habilidades de comunicação, empatia e métodos de abordagem ao paciente; fornecer aos acadêmicos a possibilidade de realizar atividades extracurriculares em ambiente hospitalar de forma descontraída e dinâmica; fomentar o trabalho em equipe, entrosamento entre colegas e união para promover o bem estar alheio e promover a alegria, entretenimento e conforto ao ambiente hospitalar.

De forma geral, para a realização das visitas, utilizaram-se jalecos de mangas compridas identificados com o nome do integrante. Os narizes de palhaço foram uma peça chave para a caracterização do grupo que se somaram a outros adornos como gravatas, perucas, vestimentas e maquiagens. Em datas comemorativas como Natal e Páscoa, a caracterização específica também foi utilizada.

As atividades realizadas englobaram diferentes métodos. Com o público adulto foram realizadas visitas preconizando o diálogo e abordagens musicais, nas quais os membros de cada grupo utilizavam violão e outros instrumentos musicais, ou 
mesmo objetos dos quais poderiam se extrair sons, para complementar a batida das músicas. Já para a ala pediátrica, realizaram-se atividades na brinquedoteca do hospital, um ambiente próximo à ala pediátrica do hospital de visita, com brinquedos e livros, para auxiliar no entretenimento de crianças de variadas idades, ou mesmo nos leitos, utilizando balóes, brinquedos, adesivos, bolas de malabares, tinta para pintura na pele, lápis, violão e outros instrumentos musicais. Foram explorados diversos ramos da arte para envolver esse público, entre eles, a música, o teatro, a dança e pintura/desenho.

\section{Resultados e Discussão}

O projeto Alegromicina contribuiu para o bem-estar dos pacientes, bem como de acompanhantes, profissionais da saúde e dos próprios voluntários. A implantação de programas que utilizam o papel do palhaço e a realização de atividades lúdicas auxilia no prognóstico das doenças e tornam a experiência da hospitalização menos traumática (Medeiros, Lacerda, Souza, Lucena \& Marques, 2013). Além disso, representa um veículo de experimentação e aprimoramento das habilidades humanas, acima de tudo a amorosidade, pelos futuros médicos (Beraldi, 2011).

Reconhecida a importância da quantificação dos beneficiados por essa ação, calculou-se o número estimado de pacientes, acompanhantes e profissionais da saúde com os quais os alunos interagiram nas áreas trabalhadas, resultando em um valor médio de mais de 800 pessoas por ano.

Durante as atividades, observou-se a reciprocidade entre hospitalizados e discentes, sendo possível apurar, de forma empírica, a gratidão dos pacientes e a melhora no quadro geral ao recebimento da visita. A extensão acadêmica descrita constituiu um caminho necessário para o sistema de saúde ao considerar que esse possui como um de seus pilares o compromisso do acolhimento. Esse princípio é baseado na construção de uma relação de confiança, compromisso e vínculo além da busca de ambiência, através da criação de espaços saudáveis e acolhedores (Ministério da Saúde, 2009). Carvalho e Rodrigues (2007) em estudo sobre a eficácia do "palhaço-doutor", baseado na percepção da equipe médica e dos acompanhantes dos pacientes, receberam relatos de que esse contribuiu de forma significativa para o processo de humanização hospitalar, sendo considerado até mesmo como parte da equipe multidisciplinar do hospital.

O projeto relatado também utiliza como interação o diálogo com escuta ativa, valorizando a relação interpessoal e os anseios do paciente. Essa prática está alinhada com o que preconiza o Método Clínico Centrado na Pessoa, que está voltado não apenas para avaliar o conhecimento do médico em relação à doença, mas também a experiência vivida pela pessoa que a possui (Barbosa \& Ribeiro, 2016). Stewart (1995) demonstrou em seu estudo que o compartilhamento das decisões entre médicos e pacientes foi associado positivamente, sendo que $76 \%$ dos casos avaliados tiveram melhora significativa do quadro clínico e, em longo prazo, na saúde do paciente. Ainda que não fossem profissionais formados, os recursos utilizados pelos discentes durante as visitas funcionaram como complemento da terapêutica instituída pelos profissionais de saúde, principalmente quanto ao bem-estar do paciente, favorecendo a humanização do cuidado hospitalar.

O Alegromicina também incluiu atividades de lazer voltadas aos idosos, que possuem maior vulnerabilidade biológica e psicossocial e, consequentemente, susceptibilidade aumentada às doenças (Barbosa et al., 2017). O envelhecimento é definido como um processo gradual decorrente de uma perda funcional progressiva ao organismo, sendo um processo variável e complexo que exige atenção interdisciplinar (Nahas, 2006) e está relacionado a um maior período de internamento, com intervenções desagradáveis.

A hospitalização provoca alterações significativas na vida dos idosos, devido ao afastamento do ambiente familiar, quebra de rotinas, medo da dependência e até mesmo da morte (Jannuzzi \& Cintra, 2006). Diante disso, as atividades de recreação constituíram uma prática de intervenção capaz de minimizar os efeitos negativos, ocupar os períodos de solidão e propiciar momentos de prazer. Ademais, notou-se que o preenchimento dos momentos de inatividade aumentou a resiliência dos pacientes geriátricos. 
Nas alas de clínica médica e hemodiálise, a atuação dos acadêmicos como de ouvintes, amigos, cantores, palhaços e comediantes era responsável por momentos de alívio, sucedidos, quase sempre, por muitos agradecimentos dos pacientes aos voluntários.

Na pediatria, os acadêmicos precisaram utilizar outras ferramentas de ludicidade para entreter o público infantil, como teatro, música, contação de histórias e o próprio espaço da brinquedoteca. Os pacientes pediátricos distraíam-se e aplicavam sua criatividade - anteriormente inibida pelo ambiente hospitalar - em atividades lúdicas. Além disso, tal interação permitiu que os acompanhantes participassem desses momentos de relaxamento e distração junto com as crianças, permitindo aliviar a aflição, a angústia e a ansiedade também desses acompanhantes, tornando a presença do projeto duplamente significativa.

A utilização dessas técnicas lúdicas no período de hospitalização é efetiva para melhora do paciente, como demonstrado em estudos (Campos \& Nakasu, 2016; Martins, et al., 2020; Oliveira, Silva \& Fantacini, 2016; Paula et al., 2019; Taets, Borba-Pinheiro, Figueiredo \& Dantas, 2013; Zanini et al., 2009). Oliveira e Oliveira (2008) afirmam que deixar o brinquedo com a criança faz com que ela memorize essa interação e recorde das experiências positivas relacionadas ao brinquedo e à ação de brincar, auxiliando no enfrentamento de situações difíceis. Além disso, atividades realizadas na brinquedoteca podem colaborar com o desenvolvimento de crianças e adolescentes, por meio de brincadeiras e da imaginação, fortalecendo os aspectos psicossociais de enfrentamento a doença (Oliveira, Silva \& Fantacini, 2016).

Uma visão muito semelhante é apresentada por Berto e Abrão (2009), que mostram que o brincar é um recurso viável e adequado para complementação do período de internação e um instrumento facilitador para realização de procedimentos médicos. Desse modo, abordagens mais leves no ambiente hospitalar podem reduzir o tempo de recuperação da criança, diminuindo o custo da hospitalização (Oliveira \& Oliveira, 2008).

Com relação aos benefícios para os familiares, sabe-se que os pais se sentem mais seguros vendo que seus filhos estão com as necessidades emocionais e médicas supridas (Soares \& Zamberlan, 2001). Essa interação, por sua vez, melhora a relação da criança com a equipe profissional, aumentando o acolhimento (Berto \& Abrão, 2009), o que culmina em uma melhor qualidade da assistência e contribui positivamente com o eixo familiar. Os benefícios da implementação da experiência aqui relatada atingiram também a equipe médica hospitalar como foi evidenciado similarmente em um estudo realizado em um hospital no município de Curitiba, Paraná, onde a utilização do brinquedo terapêutico durante o cuidado da criança facilitou o trabalho da equipe de saúde e promoveu relaxamento de tensão e distração de ambos (Araújo \& Guimarães, 2009; Cavalcanti et al., 2015; Jansen et al., 2010;).

Biologicamente, ser distraído por alguma atividade e dar risada diminui a sensação de estresse e promove a liberação de endorfina, hormônio que poderá melhorar respostas imunes e amenizar os impactos da hospitalização e do tratamento, reduzindo a exposição a estímulos dolorosos (Yim, 2016). O bom humor atua também na melhoria da circulação, fazendo com que os tecidos recebam mais oxigênio e funcionem com maior eficiência (Abreu, 2011). Dessa forma, os profissionais de saúde dos ambientes visitados, ao acompanharem a resposta emocional positiva dos pacientes durante e após as atividades, puderam constatar que o humor é importante para o desfecho favorável do binômio saúde e doença dos enfermos.

A musicoterapia utilizada pelos discentes do projeto é um eficiente recurso não farmacológico para a redução da dor, ansiedade e melhora de bem-estar, sendo testada e discutida pela literatura especializada (Campos \& Nakasu, 2016; Martins, et al., 2020). Comprova-se que há uma diminuição estatística significativa na pressão arterial de indivíduos com hipertensão no estágio 1, no nível de estresse e na ansiedade, após o programa de musicoterapia (Campos \& Nakasu, 2016; Taets et al., 2013; Zanini et al., 2009).

Quanto aos benefícios para os estudantes voluntários, a significativa contribuição do projeto se deu em vários aspectos. No decorrer da iniciativa, os estudantes foram capazes de perceber o paciente para além da sua enfermidade. Isso porque, apesar de já estarem ambientados com o meio hospitalar, encontravam-se fora de sua zona de conforto. Dessa maneira, 
o altruísmo e a empatia foram exercitados a fim de alcançar a correta abordagem para cada momento dos pacientes, sendo essas experiências essenciais ao apontarem fraquezas e desafios a serem superados, visando a melhor formação dos futuros médicos.

Jha (2014), médico e pesquisador de Harvard, indagou em uma enquete feita no Twitter "O que faz um médico ser bom?", entre as respostas, lideraram empatia, ser um bom ouvinte e compaixão, reiterando a importância dessas características na prática médica e de saúde. O contato com pessoas em situação de fragilidade, por si só, é precursor de substanciais mudanças pessoais, porém, quando associado ao contato com serviços de saúde e as diferentes realidades socioeconômicas dos pacientes, tem-se um conjunto de experiências enriquecedoras. Em vista disso, foi necessário que os voluntários se reinventassem a cada novo encontro, afinal, a criança agudizada tem percepção de doença e do internamento muito diferente do idoso dialítico crônico e este do jovem pós-trauma, por exemplo.

\section{Considerações Finais}

O presente relato abordou as atividades do grupo Alegromicina, implantado na cidade de Toledo como projeto de extensão da Universidade Federal do Paraná, Campus Toledo, tendo início em 2017 e término em 2020. Através de atividades lúdicas, como musicoterapia, caracterização com uso de adereços, encenações, diálogo e escuta ativa realizou-se o acolhimento e entretenimento dos pacientes assistidos durante as visitas a pacientes hospitalizados e do setor de hemodiálise.

No decorrer das atividades foi possível perceber a importância da humanização em saúde. Houve aproximação das relações dos extensionistas entre si, com os profissionais de saúde e com cada paciente, propiciando sentimentos afetuosos e prazerosos de forma bidirecional. Com a retirada dos estudantes de suas zonas de conforto, a capacidade de percepção das fragilidades e necessidades dos pacientes em cada situação foi estimulada, gerando uma resposta benéfica tanto aos participantes quanto aos pacientes, minimizando os efeitos negativos da hospitalização e dos tratamentos. Houve benefício também para os profissionais de saúde que trabalham nos locais visitados, já que, ao presenciarem a resposta emocional positiva dos pacientes puderam absorver essa premissa para seus atendimentos, contagiados pelo clima de descontração em um ambiente até então focado na seriedade do trabalho.

Com um total aproximado de 800 pessoas ao ano impactadas pelo projeto, foi possível perceber a importância das atividades implementadas, pois o acolhimento gera melhorias no enfrentamento das enfermidades e, inconscientemente, amenizam o processo do tratamento. Sendo o bem-estar um importante fator de saúde, os benefícios relatados no estado emocional contribuem para o atendimento global ao paciente, consolidando o pilar da integralidade nos serviços de saúde. Perante a otimização do cuidado evidenciada neste relato, encoraja-se a implementação de projetos similares ao Alegromicina em outros centros educativos, pois o resultado de uma atividade como essa mostrou-se bastante significativo na comunidade impactada, bem como um importante crescimento profissional aos participantes, considerando o caráter prático da atividade que concretiza o processo educacional e promove a intersetorialização dos serviços de saúde.

Recomendam-se novos estudos qualitativos, focados na avaliação do impacto de atividades lúdicas em ambientes hospitalares, a partir da percepção dos acompanhantes de pacientes e da equipe de saúde que trabalha nos serviços comtemplados com projetos semelhantes ao Alegromicina.

\section{Agradecimentos}

Agradecemos à Pró-Reitora de Extensão e Cultura da Universidade Federal do Paraná pelo incentivo a extensão, pesquisa e bolsas concedidas, bem como as instituições de saúde campo deste projeto pelo espaço concedido e apoio as atividades. 


\section{Referências}

Abreu, G. R. F. (2011). A terapia do (bom) humor nos processos de cuidado em saúde. Revista Baiana de Enfermagem, 25(1), 69-74. https://periodicos.ufba.br/index.php/enfermagem/article/view/5062.

Adams, P., \& Mylander, M. (2002). A Terapia do Amor. Mondrian.

Araújo, T. C. C. F., \& Guimarães, T. B. (2009). Interações entre voluntários e usuários em onco-hematologia pediátrica: um estudo sobre os "palhaçosdoutores". Estudos e Pesquisas em Psicologia, 9(3), 632-647. https://doi.org/10.12957/epp.2009.9072.

Barbosa, K. T. F., Costa, K. N. F. M., Pontes, M. L. F., Batista, P. S. S., Oliveira, F. M. R. L., \& Fernandes, M. G. M. (2017). Aging and individual vulnerability: a panorama of older adults attended by the family health strategy. Texto \& Contexto - Enfermagem, 26(2). https://doi.org/10.1590/010407072017002700015

Barbosa, M. S., \& Ribeiro, M. M. F. (2016). O método clínico centrado na pessoa na formação médica como ferramenta de promoção de saúde. Revista Médica de Minas Gerais, 26(Supl 8), 216-222. http://www.rmmg.org/artigo/detalhes/2152.

Beraldi, G. (2011). A História do Clown. http://www.conteudoseducar.com.br/conteudos/arquivos/2581.pdf.

Berto, C. E. O., \& Abrão, J. L. F. (2009). A importância do brincar no contexto hospitalar: percepção e compreensão da equipe de enfermagem. Revista de Psicologia da Unesp, 8(2), 154-157. https://seer.assis.unesp.br/index.php/psicologia/article/view/1029.

Campos, L. F., \& Nakasu, M. V. (2016). Efeitos da Utilização da Música no Ambiente Hospitalar: revisão sistemática. Revista Sonora, 6(11), 9-19. https://www.publionline.iar.unicamp.br/index.php/sonora/article/view/686.

Carvalho, F. G. R., \& Rodrigues, P. R. G. (2007). Dr. Amoroso: um trabalho de apoio social na humanização hospitalar. Anais do XI Encontro Latino Americano de Iniciação Científica e VII Encontro Latino Americano de Pós-Graduação - Universidade do Vale do Paraíba. São José dos Campos, pp. 28852888. http://www.inicepg.univap.br/cd/INIC_2007/trabalhos/humanas/inic/INICG00320_01O.pdf.

Cavalcanti, M. E. D., Bomfim, A. M. A., Rocha, M. C. G., Porto, V. F. A., Lima, E. B., \& Mesquita, T. M. (2015). Recurso lúdico no processo de educação em saúde em crianças de escolas públicas de Alagoas: relato de experiência. Interfaces - Revista de Extensão da UFMG, 3(1), 117-121. https://periodicos.ufmg.br/index.php/revistainterfaces/article/view/18969.

Crockett, Z. (2015). The Father of Pediatric Clown Care. Priceonomics. https://priceonomics.com/how-a-clown-brought-humor-to-childrens-hospitals/.

Dario, P. (2019). A relação médico-doente. Breve revisão da antiguidade à atualidade. Revista de Medicina, 98(3), 216-221. https://doi.org/10.11606/issn.1679-9836.v98i3p216-221.

Doğan, M. D. (2020). The Effect of Laughter Therapy on Anxiety, A Meta-analysis. Holistic Nursing Practice, 34(1), 35-39. https://doi.org/10.1097/hnp.0000000000000363.

Heidari, M., Borujeni, M. G., Rezaei, P., Abyaneh, S. K., \& Heidari, K. (2020). Effect of Laughter Therapy on Depression and Quality of Life of the Elderly Living in Nursing Homes. Malays J Med Sci, 27(4), 119-129. http://doi.org/10.21315/mjms2020.27.4.11.

Jannuzzi, F. F., \& Cintra, F. A. (2006). Atividades de lazer em idosos durante a hospitalização. Revista da Escola de Enfermagem da USP, $40(2), 179-187$. https://doi.org/10.1590/s0080-62342006000200005.

Jansen, M. F., Santos, R. M., \& Favero, L. (2010). Benefícios da utilização do brinquedo durante o cuidado de enfermagem prestado a criança hospitalizada. Revista Gaúcha de Enfermagem, 31(2), 247-253. https://doi.org/10.1590/s1983-14472010000200007.

Jha, A. K. (2014). What makes a good doctor, and can we measure it?. [Blog] Harvard T. H. Chan | School of public Health. https://blogs.sph.harvard.edu/ashish-jha/2014/03/20/what-makes-a-good-doctor-and-can-we-measure-it/.

Lopes, J. M. C., \& Dias, L. C. (2019). Consulta e abordagem centrada na pessoa. In Gusso, G., Lopes, J. M. C., \& Dias, L. C. Tratado de Medicina de Família e Comunidade: princípios, formação e prática. Artmed, 15, 113-123.

Luchesi, A., \& Cardoso, F. S. (2012). Terapia do Riso - Um Relato de Experiência. Revista Eletrônica da Faculdade Evangélica do Paraná, 2(1), 11-20.

Luiz, R. R. (2007). O uso do bom humor e o cuidado na saúde. Monografia de conclusão de curso, Escola Politécnica de Saúde Joaquim Venâncio.

Masseti, M. (2005). Doutores da ética da alegria. Interface - Comunicação, Saúde, Educação, 9(17), 453-458. http://dx.doi.org/10.1590/S141432832005000200026 .

Martins, E. L., Geremia, D. S., Souza, J. B., Zanettini, A., Rosa, O. M. \& Barbosa, S. S. P. (2020). Music in the hospital environment: perceptions of individuals under oncological treatment. Research, Society and Development, 9(9), e667997819. 10.33448/rsd-v9i9.7819.

Medeiros, C. M. L., Lacerda, O. R. M., Souza, I. V. B., Lucena, A. L. R., \& Marques, D. K. A. (2013). O lúdico no enfrentamento da hospitalização: percepção da família. Revista De Ciências Da Saúde Nova Esperança, 11(2), 116 - 130. https://revista.facene.com.br/index.php/revistane/article/view/501.

Ministério da Saúde. (2009). Biblioteca Virtual em Saúde do Ministério da Saúde. Dicas em Saúde: ambiência. http://bvsms.saude.gov.br/bvs/dicas/170_ambiencia.html.

Mussa, C., \& Malerbi F. E. K. (2008). O impacto da atividade lúdica sobre o bem-estar de crianças hospitalizadas. Psicologia: Teoria e Prática, 10(2), 83-93. https://www.redalyc.org/articulo.oa?id=193817427007.

Nahas, M. V. (2006). Atividade física, saúde e qualidade de vida: conceitos e sugestões para um estilo de vida ativo. Mediograf. 
Research, Society and Development, v. 10, n. 2, e53610212464, 2021

(CC BY 4.0) | ISSN 2525-3409 | DOI: http://dx.doi.org/10.33448/rsd-v10i2.12464

Nunes, G. C., Nascimento, M. C. D., \& Luz, M. A. C. A. (2016). Pesquisa Científica: conceitos básicos. Id on Line Revista de Psicologia, 10(29), 144-151. https://doi.org/10.14295/idonline.v10i1.390.

Oliveira, É. F., Silva, V. M., \& Fantacini, R. A. F. (2016). Pedagogia hospitalar: os ambientes da brinquedoteca no hospital. Research, Society and Development, 1(1), 88-104. https://doi.org/10.17648/rsd-v1i1.6.

Oliveira, R. R., \& Oliveira, I. C. S. (2008). Os Doutores da Alegria na unidade de internação pediátrica: experiências da equipe de enfermagem. Escola Anna Nery Revista de Enfermagem, 12(2), 230-236. https://doi.org/10.1590/S1414-81452008000200005.

Paula, G. K., Góes, F. G. B., Silva, A. C. S. S., Moraes, J. R. M. M., Silva, L. F., \& Silva, M. A. (2019). Estratégias lúdicas no cuidado de enfermagem à criança hospitalizada. Revista de Enfermagem UFPE On Line, 13, 1-11. https://doi.org/10.5205/1981-8963.2019.238979.

Pereira, A. S., Shitsuka, D. M., Parreira, F. J., \& Shitsuka, R. (2018). Metodologia da pesquisa científica UFSM. https://repositorio.ufsm.br/bitstre am/handle/1/15824/Lic_Computacao_Metodologia-Pesquisa-Cientifica.pdf?sequence=1.

Pines Jr., A. R., Silva, T. A. C., Lazier, T. R., Gonçalves, K. G. F., \& Leão Jr., C. M. (2013). O circo moderno: história, inovação e transição social. Fiep Bulletin, 83(Especial). http://www.fiepbulletin.net/index.php/fiepbulletin/article/view/2981.

Rodrigues, J. C., Simões, R. M. R., \& Prodocimo, E. (2019). O lúdico no ambiente da classe hospitalar: um estudo de revisão. REFACS, 7(3), 390-400. https://www.scielo.br/scielo.php?script=sci_arttext\&pid=S0080-62342011000200023.

Soares, M. R. Z., \& Zamberlan, M. A. T. (2001). A inclusão do brincar na hospitalização infantil. Revista Estudos de Psicologia, 18(2), 64-69. https://doi.org/10.1590/S0103-166X2001000200006.

Stewart, M. A. (1995). Effective physician-patient communication and health outcomes: a review. CMAJ: Canadian Medical Association Journal $=$ journal de I'Association medicale canadienne, 152(9), 1423-1433.

Taets, G. G. C., Borba-Pinheiro, C. J., Figueiredo, N. M. A., \& Dantas, E. H. M. (2013). Impacto de um programa de musicoterapia sobre o nível de estresse de profissionais de saúde. Revista Brasileira de Enfermagem, 66(3), 385-390. https://doi.org/10.1590/S0034-71672013000300013.

Yim, J. E. (2016). Therapeutic benefits of laughter in mental health: a theoretical review. The Tohoku Journal of Experimental Medicine, 239(3), 243-249. https://doi.org/10.1620/tjem.239.243.

Zanini, C. R. O., Jardim, P. C. B. V., Salgado, C. M., Nunes, M. C., Urzêda, F. L., Carvalho, M. V. C., Pereira, D. A., Jardim, T. S. V., \& Souza, W. K. S. B. (2009). O efeito da musicoterapia na qualidade de vida e na pressão arterial do paciente hipertenso. Arquivos Brasileiros de Cardiologia, 93(5), 534-540. https://doi.org/10.1590/S0066-782X2009001100015. 